\title{
Hydrothermal synthesis of microscale boehmite and gamma nanoleaves alumina
}

\author{
Ye Liu ${ }^{a}$, Ding Ma ${ }^{\mathrm{a},}$, Xiuwen Han ${ }^{\mathrm{a}}$, Xinhe Bao ${ }^{\mathrm{a}, \star}$, Wiebke Frandsen ${ }^{\mathrm{b}}$, Di Wang ${ }^{\mathrm{b}}$, \\ Dangsheng $\mathrm{Su}^{\mathrm{b}}$ \\ ${ }^{a}$ State Key Laboratory of Catalysis, Dalian Institute of Chemical Physics, Chinese Academy of Sciences, \\ Dalian 116023, PR China \\ ${ }^{b}$ Department of Inorganic Chemistry, Fritz-Haber Institute of the Max Planck Society, Berlin D-14195, \\ Germany \\ * Coressponding authors. Tel.: +86411 84379253; fax: +8641184694447. \\ E-mail addresses: dma@dicp.ac.cn (D. Ma), xhbao@dicp.ac.cn (X. Bao).
}

Keywords: Nanomaterials; Morphology control; Electron microscopy; Alumina

\begin{abstract}
Novel uniform single-crystal boehmite leaf-like nanosheets with high anisotropy (with a lateral size of $(4.5 \pm 0.5 \mu \mathrm{m}) \times(9.0 \pm 1.0 \mu \mathrm{m})$ and a thickness of $60-90 \mathrm{~nm})$ and flower-like superstructures consisting of single-crystal petals were synthesized for the first time by a simple hydrothermal method. After calcination, those boehmite structures can be transformed into single-crystal gamma-alumina nanostructures while keeping their morphology. The morphologies and the crystal structures of single crystal boehmite nanoleaves and flower-like superstructures were characterized by X-ray powder diffraction (XRD), field-emission scanning electron microscopy (FE-SEM), transmission electron microscopy (TEM), and high resolution transmission electron microscopy (HRTEM). The formation mechanism of the nanoleaves and flower-like superstructure is also discussed. The synthesis of uniform single-crystal boehmite and $\gamma$-alumina is highly helpful to study various properties of these anisotropic structures.
\end{abstract}

\section{Introduction}

Nanostructures with controlled dimensionality and morphology are of increasing interests in the field of material chemistry. Some of those interesting nanostructures are the building blocks for the new bottomup approaches to various superstructures [1]. In addition to the extensive studies carried out on zero/onedimensional nanostructures in the past decade [2], growing interests have been poured into the study of two-dimensional nanostructures. Because of high anisotropy in structure, some unique properties were observed on those two-dimensional nanostructures (nanosheets). The comparatively more developed lateral dimension of the 2D structure produces a huge amount of surface atoms arranged twodimensionally in single-crystal order. This property makes some nanosheets as promising high performance catalysts [3] and may provide new clues for various reactions at surface and interface localities [4]. They can also be used as building blocks to assemble layer-by-layer into some highlyordered superstructures [5], pillared structure with well developed mesoporosity [6], and functional nanostructures for various exciting applications [7,8]. Boehmite and its dehydrated alumina form are extensively used for preparation of various optical, electronic devices and catalysts [9-11]. However, it has been proven difficult to synthesize these materials with good crystallinity in aqueous solutions due to the very fast hydrolysis rates of alumina precursors [12], therefore the reports on alumina nanostructures with well-resolved morphologies are limited [13-16]. The synthesis of 2-D boehmite or alumina nanosheets (thickness under $100 \mathrm{~nm}$ ) with uniform morphology and their assembled superstructures have not been reported so far. Moreover, despite the industrial significance of $\gamma$-alumina, controversy still 
exists over the determination of its structure mainly due to the poor crystallinity of $\gamma$-alumina [17]. For this reason, the synthesis of uniform single-crystal $\gamma$-alumina will also be highly expected.

In this work, novel single-crystal leaf-like boehmite nanostructures (thickness under $100 \mathrm{~nm}$ ) with high anisotropy and boehmite flowers with single-crystal petals were obtained for the first time. After careful calcination, single-crystal $\gamma$-alumina nanoleaves with high anisotropy and $\gamma$-alumina flowers with single crystal petals were obtained successfully. It provides an inexpensive yet simple method to prepare boehmite or $\gamma$-alumina single-crystal nanoleaves and superstructures organized with those nanoleaves.


Fig. 1. (A) XRD patterns of the as synthesized boehmite (black) and JCPDS card No. 21-1307 (red); (B) XRD patterns of the calcinated sample (black) and JCPDS card No. 10-0425 (red). (For interpretation of the references to colour in this figure legend, the reader is referred to the web version of this article.)

\section{Experimental}

Typically, $0.96 \mathrm{~g} \mathrm{NaOH}$ was dissolved in $6 \mathrm{ml}$ deionized water. $10 \mathrm{ml}$ of $0.6 \mathrm{M} \mathrm{AlCl}_{3}$ solution was added slowly to the $\mathrm{NaOH}$ solution under drastic stirring for $10 \mathrm{~min} .20 \mathrm{ml}$ of $0.05 \mathrm{M}$ CTAB solution (in ethanol) was subsequently added dropwise into the mixture. After another $24 \mathrm{~h}$ stirring, the resulted milky mixture was transferred into a Teflon-lined stainless steel autoclave at $160{ }^{\circ} \mathrm{C}$ for $12 \mathrm{~h}$. After cooling to room temperature, the final product was collected by centrifugation, washed with ethanol, and then vacuum-dried at room temperature for $12 \mathrm{~h}$. As-obtained product was further calcined at $600{ }^{\circ} \mathrm{C}$ for $4 \mathrm{~h}$ for phase evolution study.

$\mathrm{X}$-ray powder diffraction (XRD) patterns were recorded using a Rigaku D/max-2500/PC diffractometer with $\mathrm{CuK} \alpha$ radiation $(\lambda=0.15406 \mathrm{~nm})$. Scanning electron microscope (SEM) images were obtained on a Hitachi S4800 field-emission scanning electron microscope. Transmission electron microscope (TEM) images and selected-area electron diffraction (SAED) pattern were taken with a Philips CM 200 LaB6 transmission electron microscope. High-resolution transmission electron microscope (HRTEM) images were obtained on a Philips CM 200 FEG microscope.

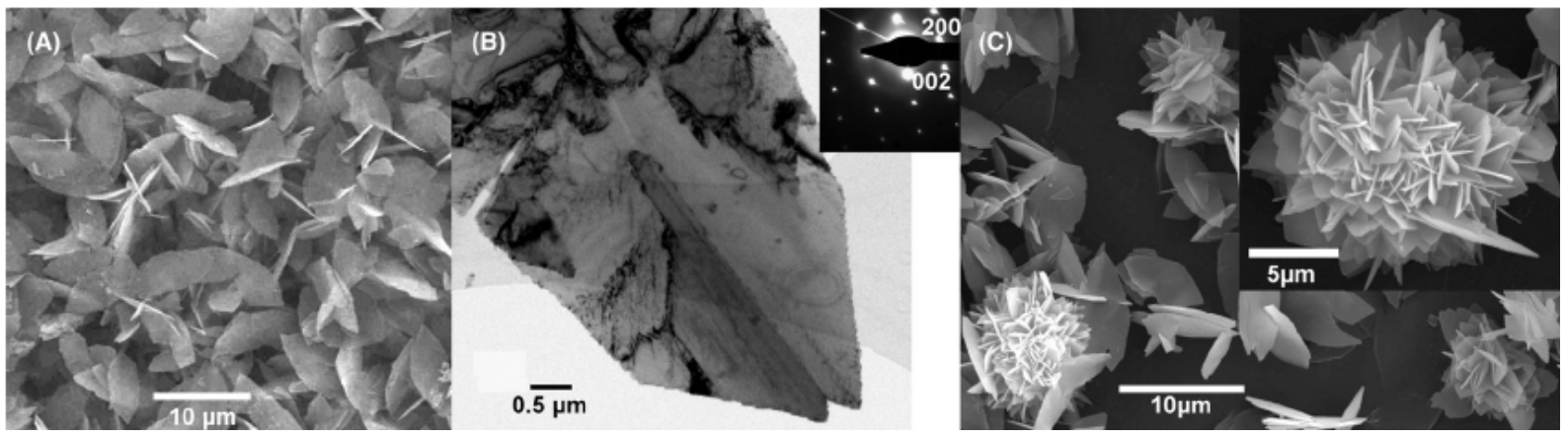

Fig. 2. (A) representative SEM images of boehmite nanoleaves; (B) TEM image of boehmite nanoleaves with the inset SAED pattern indicating the single-crystal nature of the structure; (C) representative SEM images of 3-D flower-like boehmite superstructures with single-crystal petals. 


\section{Results and discussion}

XRD pattern of the as-synthesized sample (Fig. 1A) can be indexed as boehmite with an orthorhombic unit cell $(a=3.700 \AA, b=12.227 \AA, c=2.868 \AA$, space group symmetry Amam(63), JCPDS PDF No. 21 1307). The very sharp peaks indicate a high crystallinity of the as synthesized product. The remarkably increased intensity of the (020) diffraction peak compared with that of standard pattern suggests the highly anisotropic crystallographic nature of the particles in the product along the [010] axis.

Fig. 2 shows the SEM and TEM images of the as-synthesized product. These boehmite nanostructures exhibit uniform leaf-like morphology with a lateral size of $(4.5 \pm 0.5 \mu \mathrm{m}) \times(9.0 \pm 1.0 \mu \mathrm{m})$ and a thickness of 60-90 nm (Fig. 2A). This leaf-like structure shows a preferential growth of the boehmite crystallites within the (020) plane, which explains the very strong (020) diffraction peak in the XRD pattern shown in Fig. 1A. Fig. 2B is the TEM image of a single boehmite leaf. The corresponding SAED pattern shown in the inset possesses well-resolved pattern of bright spots on [010] zone axis, indicating the single-crystal nature of the nanoleaves and a preferred growth within (020) plane. With higher concentration of $\mathrm{AlCl}_{3}$ $(0.8 \mathrm{M}$, for example) in the synthesis mixture, three-dimensional flower-like superstructures with an average diameter of about $10 \mu \mathrm{m}$ (Fig. 2C) were obtained, which are believed to be the result of an in situ assembly of the nanoleaves during crystallization process.

Furthermore, the as-synthesized product was calcined carefully in order to get its dehydrated alumina form. The XRD pattern of the calcined sample (Fig. 1B) indicates that a transformation from boehmite phase to $\gamma$-alumina after calcination occurred. All the peaks can be indexed to a cubic unit cell of $\gamma$ alumina ( $\mathrm{a}=7.900 \AA, \mathrm{b}=7.900 \AA, \mathrm{c}=7.900 \AA$, space group symmetry $\mathrm{Fd}^{-} \mathrm{m}(227)$, JCPDS PDF No.100425).

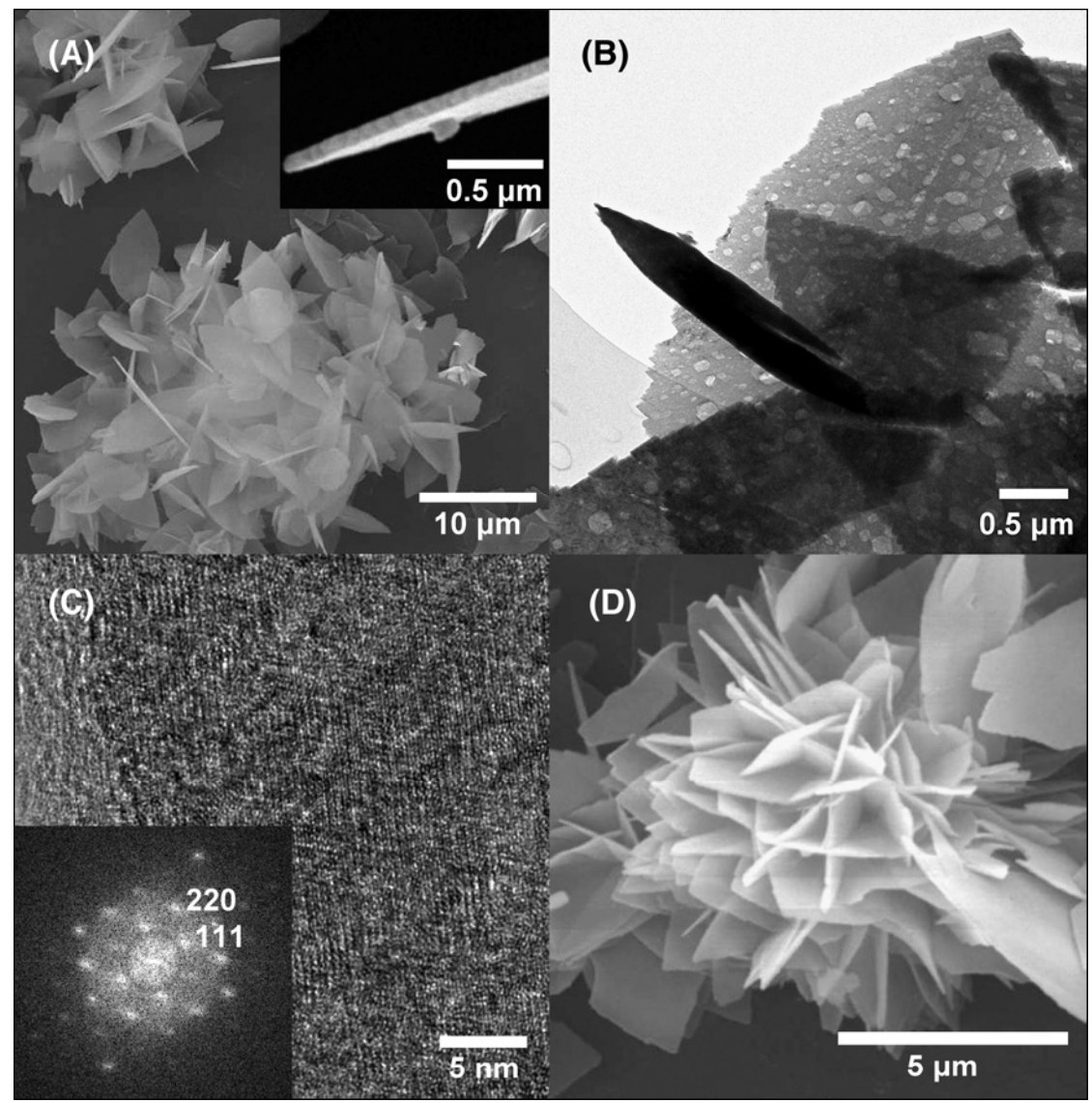

Fig. 3. (A) representative SEM images of single-crystal leaf-like $\gamma$-alumina nanosheets; (B) TEM image of single-crystal leaf-like $\gamma$-alumina nanosheets; (C) HRTEM image and FFT pattern of the simple in (A); (D) representative SEM images of 3-D flowerlike $\gamma$-alumina nanostructures. 
In addition, the SEM (Fig. 3A) and TEM (Fig. 3B) images clearly shows that the plate-like morphology of the boehmite crystallites is maintained for the alumina phase after calcination. Detailed structural information of the alumina phase is also revealed by HRTEM study. The high-resolution image (Fig. 3C) gives a d spacing of about $4.6 \AA$, which matches that of (111) crystal planes of $\gamma$-alumina very well. The corresponding FFT pattern (Fig. 3C inset) indicates a single crystal nature of the calcination-induced alumina phase. Furthermore, SEM picture (Fig. 3D) show that the morphology of the flower-like boehmite superstructure assemblies was well preserved after calcination.

In our system, the formation of boehmite $(\mathrm{AlOOH})$ can be described by the following reaction:

$\mathrm{A} 10_{2}^{-}+\mathrm{H}_{2} \mathrm{O} \rightarrow \mathrm{AlOOH}+\mathrm{OH}^{-}$

However, as the hydrolysis rate of aluminum precursor is ultra-fast in the aqueous media, most of times amorphous products or products with irregular morphologies were resulted. In order to avoid this, ethanol/water mixed solvent was used in the current synthesis, where the addition of ethanol could effectively slow down the crystal growth of boehmite, and therefore, leading to a better control over the reaction. The addition of CTAB is also critical for the successful production of those uniform boehmite nanosheets. It was reported that without using the surfactant in a similar synthetic system, only particles with random size and morphology distribution were obtained [18], which is confirmed by our observation as well (result not shown here). As is well known, boehmite is a type of layered materials, with each layer composed of parallel double chains of $\mathrm{AlO}_{6}$ octahedra, forming $\mathrm{AlOOH}$ layers with the $\mathrm{OH}$ groups sticking into the interlayer gallery. The hydroxyl groups on both surfaces of the layers form strong hydrogen bonds between the layers, holding together the layers into an ordered laminated structure [19]. In our system, the bonding between $\mathrm{CTAB}$ molecules with surface hydroxyl groups of each $\mathrm{AlOOH}$ layer effectively reduces the inclination of interlayer stacking through hydrogen bonds, which makes the growth of crystals unfavorable in c direction. In addition, the CTAB chains on the AlOOH surfaces make these particles hydrophobic on both surfaces, which block the channel for stacking and growth of the hydrophilic $\mathrm{AlO}^{-}$colloid particles over those surfaces. As a result, the interlayer crystal growth mode is remarkably inhibited, and the preferential crystal growth along the lateral direction of the AlOOH layers makes a highly anisotropic single crystal nanosheet structure of the boehmite product (Fig. 4). Thus, both the solvent system and the use of blocking agent are critical for the formation of those uniform boehmite nanosheets.

Under higher precursor concentration, more nucleation centers were produced. Aggregation of the crystallites is energetically favored since the formation of larger crystals can greatly reduce the interfacial energy of primary nanoparticles. However, by using surfactants, the crystal growth is manipulated and random aggregation might be replaced by the ordered and oriented assembly of the nanosheets [20]. Therefore, three-dimensional flower-like superstructure with single-crystal petals building blocks was obtained, as shown in Fig. 2C.

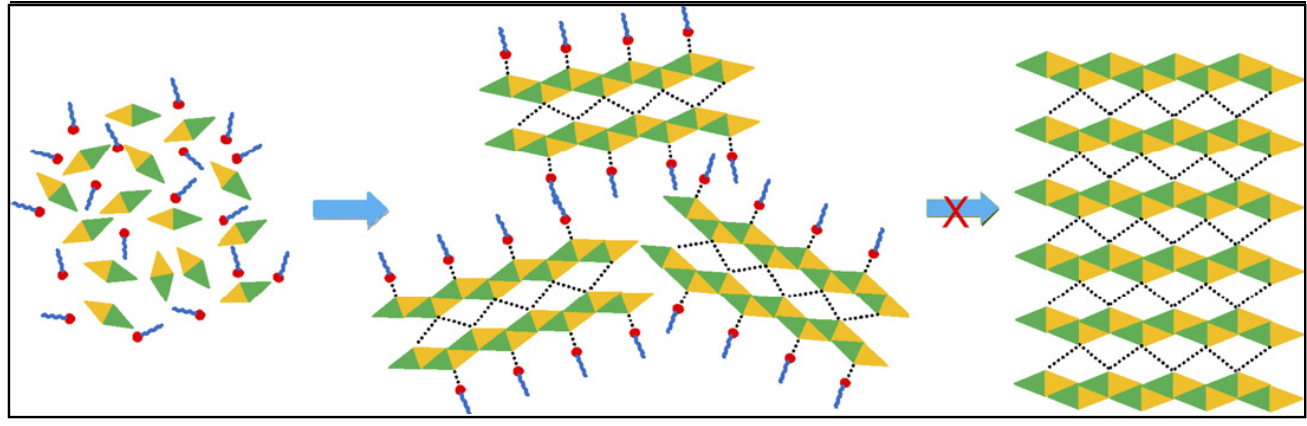

Fig. 4. The scheme for the formation of boehmite nanosheets. 
In summary, using a simple surfactant-assisted hydrothermal method and low-cost raw materials, uniform single-crystal leaf-like boehmite nanosheets (thickness under $100 \mathrm{~nm}$ ) with high anisotropy and 3-D flower-like boehmite superstructures with single-crystal petals are obtained for the first time. After calcination, single-crystal leaf-like $\gamma$ - alumina nanosheets with high anisotropy and 3-D flower-like $\gamma$ alumina nanostructures with single-crystal petals are obtained. The synthesis of uniform single-crystal boehmite and $\gamma$-alumina is highly helpful to study various properties of these anisotropic structures.

Acknowledgments

We are grateful for the support of National Natural Science Foundation of China (No. 90206036) and the Ministry of Science and Technology of China (G1999022406). The authors thank Gang Hu (Oxford University) for the helpful discussion.

\section{References}

[1] A.P. Alivisatos, Science 271 (1996) 933.

[2] M. Law, J. Goldberger, P.D. Yang, Annu. Rev. Mater. Res. 34 (2004) 83.

[3] A. Takagaki, M. Sugisawa, D. Lu, J.N. Kondo, M. Hara, K. Domen, S. Hayashi, J. Am. Chem. Soc. 125 (2003) 5479.

[4] A.I. Khan, L. Lei, A.J. Norquist, D O'Hare, Chem. Commun. (2001) 2342.

[5] G. Hu, D. O'Hare, J. Am. Chem. Soc. 127 (2005) 17808.

[6] L. Wang, Y. Ebina, K. Takada, K. Kurashima, T. Sasaki, Adv. Mater. 16 (2004) 1412.

[7] E. Hosono, S. Fujihara, I. Honma, H. Zhou, Adv. Mater. 17 (2005) 2091.

[8] J. Matsui, M. Mitsuishi, A. Aoki, T. Miyashita, J. Am. Chem. Soc. 126 (2004) 3708.

[9] I.S. Park, M.S. Kwon, N. Kim, J.S. Lee, K.Y. Kang, J. Park, Chem. Commun. (2005) 5667.

[10] Y. Kim, S.M. Lee, C.S. Park, S.I. Lee, M.Y. Lee, Appl. Phys. Lett. 71 (1997) 3604.

[11] E.P. Gusev, M. Copel, E. Cartier, I.J.R. Baumvol, C. Krug, M.A. Gribelyuk, Appl. Phys. Lett. 76 (2000) 176.

[12] H.J. Kim, H.C. Lee, C.H. Rhee, S.H. Chung, H.C. Lee, K.H. Lee, J.S. Lee, J. Am. Chem. Soc. 125 (2003) 13354.

[13] P.A. Buining, H.N.W. Lekkerkerker, J. Phys. Chem. 97 (1993) 11510.

[14] D. Kuang, Y. Fang, H. Liu, C. Frommen, D. Fenske, J. Mater. Chem. 13 (2003) 660.

[15] P. Gao, Y. Xie, Y. Chen, L. Ye, Q. Guo, J. Cryst. Growth 285 (2005) 555.

[16] H.Y. Zhu, X.P. Gao, D.Y. Song, S.P. Ringer, Y.X. Xi, R.L. Frost, Microporous Mesoporous Mater. 85 (2005) 226.

[17] G. Paglia, C.E. Buckley, A.L. Rohl, B.A. Hunter, R.D. Hart, J.V. Hanna, L. T. Byrne, Phys. Rev., B 68 (2003) 144110 .

[18] S. Music, D. Dragcevic, S. Popovic, N. Vdovic, Mater. Chem. Phys. 59 (1999) 12.

[19] B.C. Lippens, J.H. De Boer, Acta Crystallogr. 17 (1964) 1312.

[20] Z.P. Zhang, H.P. Sun, X.Q. Shao, D.F. Li, H.D. Yu, M.Y. Han, Adv. Mater. 17 (2005) 